\title{
Adrenal Medulla Neoplasm
}

National Cancer Institute

\section{Source}

National Cancer Institute. Adrenal Medulla Neoplasm. NCI Thesaurus. Code C4856.

A benign or malignant (primary or metastatic) neoplasm affecting the adrenal medulla. 